\title{
Effectiveness of Disinfection of Anesthetics Tubes in Oral Surgery-An in Vitro Study
}

José Kayque Neves ${ }^{1}$, Mayke Felipp de Araújo Martins², Josefa Elaine Silva Germinio ${ }^{2}$, Maria Cristina de Andrade $^{1}$ and Sibele Ribeiro de Oliveira ${ }^{2}$

1. Dentistry course, Research Group on Pathology of Communicable and Non-Communicable Diseases (GPPATO), Centro Universitário Tabosa de Almeida/Asces-Unita, University district, Caruaru/Pernambuco 55016-901, Brazil

2. Biomedicine course, Research Group on Pathology of Communicable and Non-Communicable Diseases (GPPATO), Centro Universitário Tabosa de Almeida/Asces-Unita, University district, Caruaru/Pernambuco 55016-901, Brazil

\begin{abstract}
Context: Anesthetic tubes are always on the surgical table, for this reason, it's necessary to perform its disinfection, generally by chemicals. Aim: Was to analyze the effectiveness of tubes decontamination made by disinfectant substances used in a clinical setting and to identify the microorganisms found on their surface. Materials and Methods: 14 anesthetic tubes were collected from dental clinic, on 5 groups. 01 plastic and 01 glass tubes that were not submitted to any disinfection process composed the control group and they had been sown in Agar Blood. Experimental groups underwent immersion and fixation with the proposed chemicals for 1 minute, laminar flow hood was used to seed it in Agar Blood and then it was transferred to incubator for 24 hours at $37^{\circ} \mathrm{C}$. Tests were used to identify the main Gram positive pathogenic genera. Gram-negative pathogenic colonies isolated were seeded in biochemical means of identification. Results: It was possible to identify the presence of Staphylococcus coagulase negative, Staphylococcus aureus, Streptococcus viridans, Staphylococcus saprophyticus, Escherichia coli and Acinetobacter spp, as well as the effectiveness of chemical solutions proposed in this study. Conclusions: All chemical solutions proposed in this study were effective for the surface disinfection process of anesthetic tubes.
\end{abstract}

Key words: Anesthetics, antimicrobial effect, disinfection, oral surgery, microbiology.

\section{Introduction}

One of the biggest problems faced by the specialists of oral surgery clinics and dental clinics is about secondary infection, among other things, due to inadequate practice in the steps to be followed to maintain an aseptic chain during procedures performed [1]. The tubes containing anesthetic solution is a consumables that have been always presented on the surgery table, being necessary to be decontaminated on its surface. This procedure is routinely performed by chemicals such as: Chlorhexidine 2\%, Povidone 10\% and Ethyl alcohol $70 \%[2,3]$.

It is essential that dental professionals follow criteria to ensure patient safety in the prevention of

Corresponding author: Sibele Ribeiro de Oliveira, Ph.D., research fields: microbiology. possible contamination. Most of the current validated protocols are easy to understand, also they have a low cost and require a minimum time for execution. However, lots of them are not properly followed because the lack of awareness of some professionals [4-6].

In addition, it has been followed by standards for infection control, in view of reducing significantly the risk of contamination in the dental clinics, contributing in transmission controlling and reducing patient exposure to pathogenic microorganisms [4, 6-8].

It is essential to keep the vigilance regarding fundamental pipelines ensuring blocking the transmission of pathogenic microorganisms, preventing cross-contamination. The inappropriate behavior of some careless professionals, to the 
referred biosecurity protocols can enlarge the risk of secondary infection [9].

Biosecurity should be seen as a summation of knowledge, habits and behaviors, which must be incorporated by society, so that professionals may develop it with more security incorporating into the communication and perception of risk in social sectors [10].

Throughout the health care field, the transmission of infectious microorganisms led to the increasing concern about the effective use of disinfectants and antiseptics. This approach is particularly observed in dentistry office, in many instruments and environmental surfaces become contaminated with saliva and blood during routine procedures [11].

There are multiple microorganisms with high contamination potential in the dental office, among which are highlighted: Staphylococcus aureus, Staphylococcus epidermidis, Streptococcus mutans, Streptococcus mitis, Staphylococcus haemolyticus, Brevundimonas spp., Streptococccus salivarius, Corynebacterium diphtheriae, Bacteroides fragilis, Staphylococcus warneri and Peptoestreptococos. Besides these, the existence of Escherichia coli and Enterococcus faecium in dental offices is an indicator of fecal contamination [12-15].

Because of the importance of the issue, the main purpose of this study was to analyze the effectiveness of surface decontamination of anesthetic tubes submitted to disinfectant substances routinely used to decontaminate objects in dental clinics, as well as to phenotypically identify the most frequently microorganisms found in surfaces, by microbiological testing in vitro.

\section{Methods and Material}

This study, made in laboratory, in an observational and descriptive way, was conducted in dental clinics of the ASCES College, located in the city of Caruaru-PE, Brazil. It collected 14 tubes containing anesthetic solution, 07 plastic tubes and 07 glass tubes, subsequently packaged in sterile assay tubes, kept tightly closed and transported immediately to the microbiology laboratory. The assay tubes transported from a dental office, were divided into 4 groups: plastic tubes for friction disinfection, glass tubes for friction disinfection, plastic tubes for immersion disinfection and glass tubes for immersion disinfection, and the tests were conducted in triplicate. The control group was consisted by 01 plastic tube and 01 glass tube which have not been subjected to disinfection methods (Fig. 1).

All anesthetic cartridges were inoculated by rolling technique in Agar Sheep Blood. Except for the control group, other anesthetic tubes were submitted to immersion and friction methods with the chemicals proposals: Chlorhexidine $2 \%$, Povidone $10 \%$ and Ethyl alcohol 70\%, during a period of 1 minute, all procedures were performed in a laminar flow hood. The inoculated plates were transferred to a bacteriological incubator from 18 to 24 hours, average temperature of $35-37{ }^{\circ} \mathrm{C}$, after they were analyzed about the presence or absence of phenotypic laboratory, to identify the gender and, when possible, the species. For this, Gram staining technique was used, seeding in selective culture media and differential, as well as in complementary biochemical tests, that allow the microorganism metabolism viewing interest pathogenic microbial growth. Positive samples, for the presence of the microorganism Staphylococcus spp. were used into the catalase test, the DNase culture and also examined the sensitivity or resistance to novobiocin. In the search for Gram-positive bacteria of the genus Streptococcus spp., it was observed the type of hemolysis, from which complementary testing was used to identify species of dental interest. For Gram-negative bacteria isolated, colonies were seeded in the following biochemical identification means: TSI (Triple Sugar Iron), SIM (Sulfito, Indol e Motilidade), Simmons of Citrate and Urea of Christensen. The oxidase test was used for analysis of 


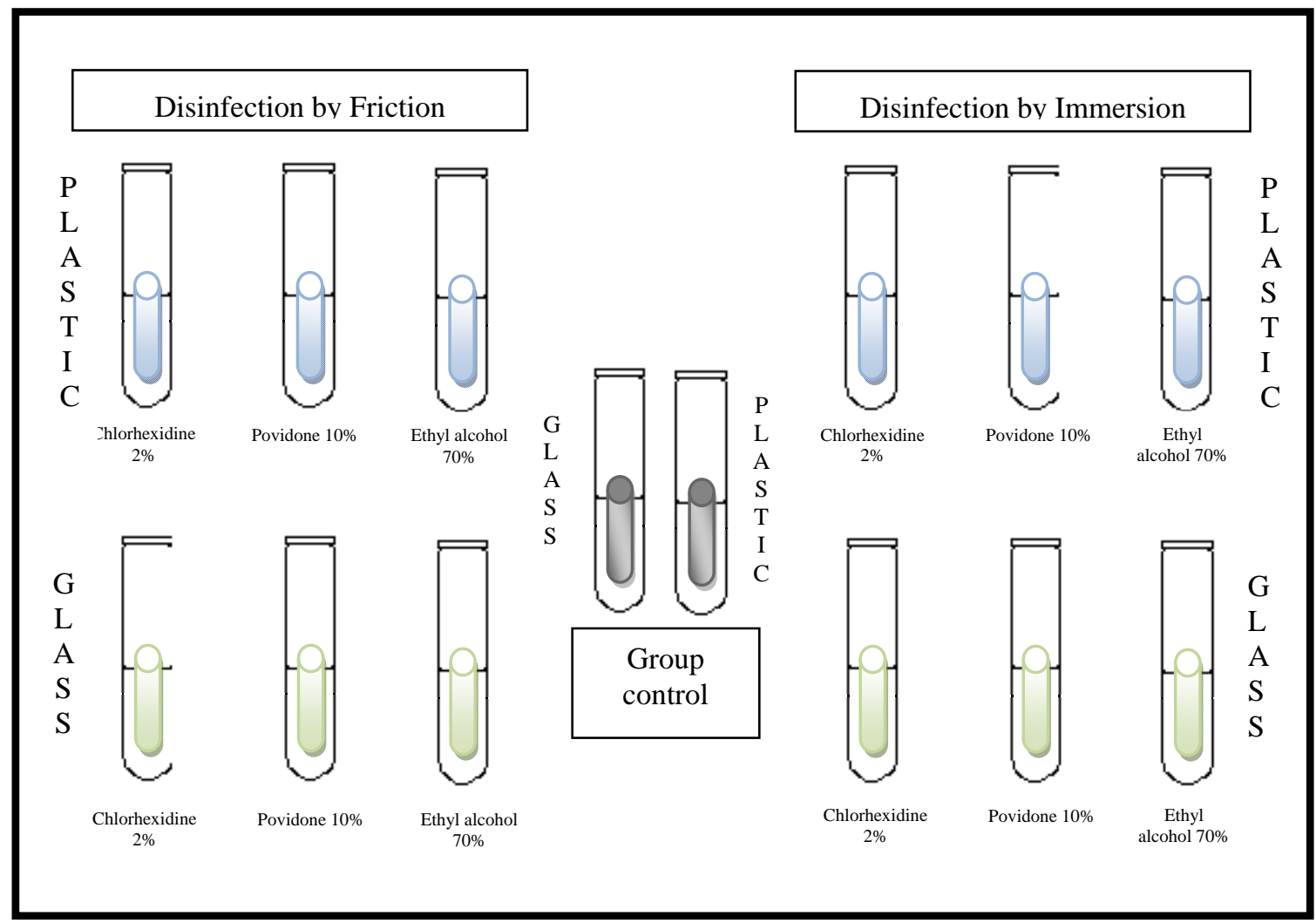

Fig. 1 Scheme representing the distribution of anesthetic tubes for microbiological procedures.

Table1 Microorganisms isolated in anesthetic tubes in control group.

\begin{tabular}{|l|l|}
\hline Microbial Classification & Genus and/or bacterial species found \\
\hline Gram Positive Bacteria & $\begin{array}{l}\text { Staphylococcus coagulase Negativa, Staphylococcus aureus, Streptococcus viridans, } \\
\text { Staphylococcus saprophyticus. }\end{array}$ \\
\hline Gram Negative Bacteria & Escherichia coli, Acinetobacter spp. \\
\hline
\end{tabular}

bacilli Gram-negative non-fermenting glucose levels.

\section{Results}

Before the submission of anesthetic tubes to disinfection procedures by immersion and friction methods, the surface of anesthetic tubes were isolated, 06 kinds of microorganisms, which are identified according to their morphology, Gram positive species of the genus Staphylococcus spp. and Streptococcus spp., as well as Gram negative fermenters and non-fermenters Glucose (Table 1).

After immersion and fixation made by the disinfectant solutions proposed in this study during 1 minute and then incubated in the same culture media recommended, it was found that both techniques (immersion and friction) associated with the
Chlorhexidine 2\%, Povidone 10\% and Ethyl alcohol $70 \%$, proved effectiveness, disinfecting the surface of anesthetic tubes, either plastic or glass surface.

\section{Discussion}

The diagnostic of microorganisms in periodontal diseases has been greatly highlighted, considering that these pathologies has presented, in most cases, with the presence of microorganisms. The main source of aggression to periodontal structures is the complexity of normal microorganisms living in the oral cavity, particularly the gingival sulcus [13]. Beyond the oral microorganisms, which live there, external microorganisms might interfere, causing infection in lots of dental procedures, whether invasive or not. This can be aggravated by the lack of appropriate care 
in handling materials used in various processes as well as the use of anesthetic tubes.

Agreeing with the study of Sthephens et al. [12], who mentioned possible microorganisms contamination indicators in dental office as the species Staphylococcus aureus, Staphylococcus coagulase negativo, Streptococcus mutans, Streptococcus mitis, Streptococccus salivarius, Corynebacterium diphtheriae, Bacteroides fragilis, Peptoestreptococos and also the presence of Escherichia coli, in the dental environment as an indicator of fecal contamination, this research has revealed the presence of Staphylococcus aureus, Staphylococcus negative coagulase and Escherichia coli in anesthetic tubes, found previously in disinfection process.

The present study has compared the techniques routinely used for disinfection of surface anesthetic tubes ranging in fixation and immersion techniques. To Malamed [16] the friction technique is more suitable to carry out disinfection, and the immersion technique may be diffusion of solution into the anesthetic tubes by semipermeable diaphragm.

These results show that all disinfectant solutions proposed (Chlorhexidine 2\%, Povidone 10\% and Ethyl alcohol 70\%) were effective within one minute of disinfection on anesthetic tubes. The analysis of Jarral et al. [17] both solutions promote immediate reduction in the number of bacteria, however, Chlorhexidine has more effective reduction. Tanner et al. [18] compared with Chlorhexidine and Polyvinylpyrrolidone in surgical interventions, with regard handwashing, showing that microbiological results after the use of chlorhexidine, were more effective than with Polyvinylpyrrolidone about reducing the number of Colony Forming Units (UFCs) both immediately, washing during 2 hours, after wash at the end of the surgery. Gonçalves et al. [19] in their systematic review, concluded that there is safe use of alcoholic preparation for surgical antisepsis of hands, however, he emphasized that the effectiveness of alcohol depends on its concentration and contact time.
The fact of this study has highlighted the presence of Gram Positive Staphylococcus coagulase negative, Staphylococcus aureus, Streptococcus viridans and Staphylococcus saprophyticus in anesthetic tubes before the disinfection process, and fortified the importance of this disinfection process, prior to dental procedures using anesthetic tubes.

Barreto et al. [5] reported about assessing the degree and extent of contamination generated in the environment of a dental clinic, during procedure with ultrasound device. After exposure petri dishes containing agar BHI (Brain Heart Infusion) supplemented with sheep blood (5\%), arranged in five specific locations close to the patient, there was a significant difference in the degree of contamination between different areas analyzed and the genus Staphylococcus spp. predominated in the samples, making up more than half of the total species found. In the case of Gram Negative found in this study, Escherichia coli and Acinetobacter spp., was related the same about the failure of various procedures in dentistry. Pereira et al. [20], has analyzed the antimicrobial capacity of 8 dental cements used for cementation on bacterial cultures of Streptococcus mutans, Streptococcus pyogenes, Staphylococcus aureus, Enterococcus faecalis and Escherichia coli. It was shown that only the $E$. coli is resistant before all evaluated cementing agents, demonstrating their viability even after the disinfection procedures performed, may, this ineffectiveness, be linked to the fact that the chemical composition of the cell wall of Gram negative bacteria differs from Gram positive bacteria.

Acinetobacter spp., also mentioned in this study, has been a major challenge for dentistry, when it refers to patients with serious systemic conditions hospitalized in Intensive Care Units (ICUs). Barbosa et al. [21] isolated Acinetobacter baumannii, Escherichia coli and other gram-negative organisms in blood cultures and tracheal secretion of 73 patients in the ICU. It is noteworthy that Gram-negative bacteria 
are not the most common in the microflora of the mouth, however, it occurs when changing the oral environment due to biofilm accumulation and development of periodontal disease. The species Staphylococcus aureus, Escherichia coli and Acinetobacter baumannii, in this study, in addition to Streptococcus pneumoniae, Haemophilus influenzae, Pseudomonas aeruginosa, Streptococcus hemolyticus and Enterobacter spp., mentioned by Barbosa et al. [21], Gomes, Esteves [22] and Xie et al. [23] belong to oral microbiota of patients ICU.

It is possible that during the execution of some dental procedures in the ICU decubitus, there is a need to use local anesthetics [16, 24]. In this study, it was found that the presence of microorganisms of the species Staphylococcus aureus, Escherichia coli and Acinetobacter spp., which have a high level of contamination of these patients ICU [21], highlights the need for disinfecting the surface of anesthetic tubes before using.

It is expected that this study may contribute to future research allowance that addresses protocols for disinfection of surface critical materials directed to oral surgery, as well as, in specialties that trigger a high risk of cross-contamination, seeking to provide greater patient safety and create opportunities of better working conditions to the dental professional.

\section{Conclusions}

Microbiological aspects have become increasingly important in current dentistry, especially when related to invasive procedures.

In general, there is a need for disinfection to prevent recurrent infections caused by possible pathogens identified on the surface of anesthetic tubes used in dentistry.

All chemical solutions proposed in this study (Chlorhexidine 2\%, Povidone 10\% and Ethyl alcohol $70 \%$ ) were effective for the surface disinfection process of anesthetic cartridges, both the friction method and immersion.

\section{Conflicts of Interest}

There are no conflicts of interest to declare.

\section{Acknowledgements:}

Thanks to the Microbiology Laboratory of the Tabosa of Almeida University for the availability of use of the facilities as well as the financial support.

\section{References}

[1] Orzechowska-Wylegala, B., Wylegala, A., Bulinski, M., and Niedzielska, I. 2015. "Antibiotic Therapies in Maxillofacial Surgery in the Context of Prophylaxis." BioMed Research International: 819086.

[2] Hupp, J. R., Ellis, III E., and Tucker, M. R. 2014. Contemporary Oral and Maxillofacial Surgery. St. Louis: Elsevier Health Sciences.

[3] Chidambaranathan, A. S., and Balasubramanium, M. 2017. "Comprehensive Review and Comparison of the Disinfection Techniques Currently Available in the Literature.” Journal of Prosthodontics: 1-8.

[4] Pinto, K., and Paula, C. 2003. "Biosecurity Protocol at Dental Office: Time and Cost.” Rev biociênc. 9 (4): 19-23.

[5] Barreto, A. C. B., Vasconcelos, C. P. P., Girão, C. M. S., Rocha, M. M. N. P., Mota, O. M. L., and Pereira, S. L. S. 2011. "Environmental Contamination by Aerosols during Treatment Using Ultrasonic.” Braz J Periodontol. 21 (2): 79-84.

[6] Sebastiani, F. R., Dym, H., and Kirpalani, T. 2017. “Infection Control in the Dental Office.” Dent Clin N Am. 61: 435-57.

[7] Pinelli, C., Garcial, P. P. N. S., Campos, J. A. D. B., Dotta, E. A. V., and Rabello, A. P. 2011. "Biosecurity and Dentistry: Beliefs and Attitudes among Dental Students Regarding Infection Control.” Saúde Soc. 20 (2): 448-61.

[8] Paiva, P. V. F., Machado, L. S., Valença, A. M. G., and Moraes, R. M. 2013. “A Proposal of Serious Game for Teaching Biosecurity in Dentistry.” Pesq Bras Odontoped Clin Integr. 13 (2): 135-9.

[9] Pimentel, M. J., Batista Filho, M. M. V., Santos, J. P., and Rosa, M. R. D. 2012. "Biosecurity: Behavior of Dental Students in Control of Cross Infection.” Cad Saúde Colet. 20 (4): 525-32.

[10] Hinrichsen, S. L. 2013. Biosecurity and Infection Control (2th ed.). Rio de Janeiro: Guanabara Koogan.

[11] Yagiela, J. 2011. A Pharmacology and Therapeutics for Dentist (6th ed.). Rio de Janeiro: Guanabara Koogan. 
[12] Stephens, J., Kinger, R., and Kettering, J. 1994. “In Vitro Comparison of the Effectiveness of Three Surface Disinfectants.” Col Dent J. 22 (6): 40-6.

[13] Uzeda, M. 2002. Oral Microbiology: Caries Etiology, Periodontal Disease and Endodontic Infections. Rio de Janeiro: Medsi Editora.

[14] Silva, F. C., Antoniazzi, M. C. C., Rosa L. P., and Cardoso Jorge, A. O. 2003. "Study of Microbiological Contamination in Radiographic Equipment." Rev biociênc. 9 (2): 35-43.

[15] Westerway, S. C., Basseal, J. M., Brockway, A., Hyett, J. A., and Carter, D. A. 2017. "Potential Infection Control Risks Associated with Ultrasound Equipment-A Bacterial Perspective.” Ultrasound Med Biol. 43 (2): 421-426.

[16] Malamed, S. F. 2013. Handbook of Local Anesthesia (6th ed.). St. Louis: Elsevier Health Sciences.

[17] Jarral, O. A., Mccormack, D. J., Ibrahim, S., and Shipolini, R. A. 2011. "Should Surgeons Scrub with Chlorhexidine or Iodine Prior to Surgery?” Interact Cardiovasc Thorac Surg. 12 (6): 1017-21.

[18] Tanner, J., Dumville, J. C., Norman, G., and Fortnam, M. 2016. "Surgical Hand Antisepsis to Reduce Surgical Site Infection.” Cochrane Database Syst Rev. 1: CD004288.

[19] Gonçalves, K. J., Graziano, K. U., and Kawagoe, J. Y.
2012. “A Systematic Review of Surgical Hand Antisepsis Using An Alcohol Preparation Compared to Traditional Products.” Rev Esc Enferm USP. 46 (6): 1484-93.

[20] Pereira, D. C., Afonso, T. S., and Chavasco, J. K. 1998. "In Vitro Studies of the Antimicrobial Action of Dental Cements on Streptococcus Mutans, Streptococcus Pyogenes, Staphylococcus Aureus, Enterococcus Faecalis, and Escherichia Coli." R Un Alfenas. 4 (1): 175-8.

[21] Barbosa, J. C. S., Lobato, P. S., Menezes, S. A. F., Menezes T. O. A., and Pinheiro, H. H. C. 2010. "Patients Profile under Intensive Care with Nosocomial Pneumonia: Key Etiological Agents.” Rev Odontol UNESP. 39 (4): 201-6.

[22] Gomes, S. F., and Esteves, M. C. L. 2012. "Role of the Surgeon Dentist in ICU: A New Paradigm.” Rev bras odontol 69 (1): 67-70.

[23] Xie, J., Peters, B. M., Li, B., Li, L., Yu, G., Xu, Z., and Shirtliff, M. E. 2017. "Clinical Features and Antimicrobial Resistance Profiles of Important Enterobacteriaceae Pathogens in Guangzhou Representative of Southern China, 2001-2015.” Microbial Pathogenesis 107: 206-11.

[24] Morais, T. M., and Silva, A. 2015. Fundamentals of Dentistry in Hospital Environment/ICU. Rio de Janeiro: Elsevier Health Sciences. 